\title{
Analysis of Area under the ROC Curve of Energy Detection
}

\author{
Saman Atapattu, Student Member, IEEE, Chintha Tellambura, Senior Member, IEEE, \\ and Hai Jiang, Member, IEEE
}

\begin{abstract}
A simple figure of merit to describe the performance of an energy detector is desirable. The area under the receiver operating characteristic (ROC) curve, denoted (AUC), is such a measure, which varies between $\frac{1}{2}$ and 1 . If the detector's performance is no better than flipping a coin, then the AUC is $\frac{1}{2}$, and it increases to one as the detector performance improves. However, in the wireless literature, the AUC measure has gone unnoticed. In this paper, to address this gap, we comprehensively analyze the AUC of an energy detector with nodiversity reception and with several popular diversity schemes. The channel model is assumed to be Nakagami- $m$ fading. First, the average $\mathrm{AUC}$ is derived for the case of no-diversity reception. Second, the average $\mathrm{AUC}$ is derived for diversity reception cases including maximal ratio combining (MRC), square-law combining (SLC) and selection combining (SC). Further, for Rayleigh fading channels, the impacts of channel estimation errors and fading correlations are analyzed. High SNR (signalto-noise ratio) approximations and the detection diversity gain are also derived. The analytical results are verified by numerical computations and by Monte-Carlo simulations.
\end{abstract}

Index Terms-Area under the curve, energy detection, receiver operating characteristic (ROC).

\section{INTRODUCTION}

D ETECTION of the presence or the absence of an unknown signal has recently received tremendous attention in view of the development of cognitive radio and ultrawideband (UWB) systems. Signal detection based on the received signal energy, referred to as energy detection, is a commonly used approach. The energy detector, a non-coherent detection device, measures the received signal energy over an observation time period, compares the measured energy level with a pre-defined threshold, and determines the presence or the absence of the unknown signal. Since it does not require channel gains and other parameter estimates, the energy detector might enable certain wireless devices to become low-cost.

The performance of an energy detector is traditionally characterized through its receiver operating characteristic (ROC) curves [1]. ROC curves are generated by plotting either detection probability $\left(P_{d}\right)$ versus false alarm probability $\left(P_{f}\right)$ or missed detection probability $\left(1-P_{d}\right)$ versus $P_{f}$ (called

Manuscript received July 21, 2009; revised October 28, 2009; accepted December 10, 2009. The associate editor coordinating the review of this paper and approving it for publication was G. Colavolpe.

This work was supported by the Natural Science and Engineering Research Council (NSERC) of Canada under a strategic grant, and the Alberta Ingenuity Fund (AIF), Alberta, Canada under a New Faculty Award.

The authors are with the Department of Electrical and Computer Engineering, University of Alberta, Edmonton, Alberta, Canada T6G 2V4 (e-mail: \{atapattu, chintha, hai.jiang\}@ece.ualberta.ca).

Digital Object Identifier 10.1109/TWC.2010.03.091085 complementary ROC) [2]. Extensive ROC analysis of the energy detector is available in the wireless literature. For instance, in [3], $P_{d}$ and $P_{f}$ are derived for Rayleigh, Rice and Nakagami fading channels. In [4], the analysis focuses on the no-diversity case under Rayleigh, Rice and Nakagami fading channels, and in [5] the focus is on different diversity receptions such as maximal ratio combining (MRC), selection combining (SC), switch-and-stay combining (SSC), squarelaw combining (SLC) and square-law selection (SLS) under Rayleigh fading channels. The energy detector with equal gain combining (EGC) reception under Nakagami- $m$ fading channels is analyzed in [6]. The energy detector performance is investigated in [7] for relay-based cognitive radio networks and in [8] for channels with both multipath fading and shadowing. Finally, looking further afield, we find that ROC analysis is regularly used, for example, in the health care field for diagnostic tests, drug testing and others [9], and in machine learning algorithms [10].

Detection probability $P_{d}(\lambda)$ and false alarm probability $P_{f}(\lambda)$ depend on the threshold $(\lambda)$ of the energy detector, the number $(u)$ of samples taken for the decision statistic, fading parameters of the fading channel, the number $(L)$ of diversity branches or the number $(n)$ of relays, and average signalto-noise ratio (SNR) $(\bar{\gamma})$ for each branch. When threshold $\lambda$ changes from $0 \rightarrow \infty$, the ROC curve starts at the upperright point $(1,1)$ and eventually moves to the lower-left point $(0,0)$. When $\bar{\gamma}, L$ or $n$ increases and $u$ decreases, the ROC curves are shifted to the upper left-hand side of the ROC graph [4]- [8]. Generally, ROC curves are plotted by varying only one parameter while keeping other parameters fixed, and a variety of curves can be generated for different combinations of parameters of interest.

Although the ROC curves fully characterize the performance of an energy detector, it is desirable to have a single figure of merit. Such a measure is the area under the ROC curve (AUC), which varies between $\frac{1}{2}$ and 1 . If the detector's performance is no better than flipping a coin, then the AUC becomes $\frac{1}{2}$, and it increases to one as the detector performance improves. As well, the Area Theorem [11] has shown that the AUC is a measure of the detection capability. Actually, in [12], it has been pointed out that the area under the curve represents the probability that choosing the correct decision at the detector is more likely than choosing the incorrect decision. However, as indicated in [13], [14], the exact computation of AUC is difficult for realistic detection tasks. Therefore, the previous research efforts mainly focus on bounds of the AUC [13], [14] or the asymptotic expansion 
and limiting value of AUC [15]. Unlike [13]-[15], our target is to derive closed-form expressions for the AUC of an energy detector in several scenarios that are of interest to wireless researchers.

In this paper, we comprehensively analyze the AUC of an energy detector with no-diversity reception and with several popular diversity schemes. The channel model is assumed to be Nakagami- $m$ fading. First, the average AUC is derived for the case of no-diversity reception. Second, the average AUC is derived for diversity reception cases including maximal ratio combining, square-law combining and selection combining. Further, for maximal ratio combining under Rayleigh fading (which is a special case of Nakagami- $m$ fading), the impacts of channel estimation errors and fading correlations are analyzed. High SNR approximations and the detection diversity gain are also derived. The analytical results are verified by numerical computations and by Monte-Carlo simulations.

The rest of the paper is organized as follows. The system model is described in Section II. AUC of the energy detector is analyzed in Section III. The average AUC of the energy detector with no-diversity and with diversity receptions over Nakagami- $m$ fading channels is analyzed in Section IV. The average AUC with maximal ratio combining under Rayleigh fading is considered in Section V when channel estimation errors and channel fading correlations exist. The numerical and simulation results are presented in Section VI. The concluding remarks are made in Section VII.

\section{SySTEM Model}

At an energy detector, a two-state model can be used to represent the received signal. Assuming flat fading, the received signal at the receiver at time $t$ is

$$
y(t)= \begin{cases}w(t) ; & H_{0}, \\ h s(t)+w(t) ; & H_{1},\end{cases}
$$

where $h$ is the complex channel gain of the channel between the transmitter and the receiver, $s(t)$ is the transmitted signal with average power $E_{s}$, and $w(t)$ is the additive white Gaussian noise (AWGN) signal at the receiver with the single-sided power spectral density $N_{0}$. The hypothesis $H_{0}$ means that the signal $s(t)$ is absent, while the hypothesis $H_{1}$ means that the signal $s(t)$ is present. Further, we assume that the channel is time-invariant during the detection process.

\section{A. Energy Detector}

As described in [2], [4], [5], first the energy detector uses a properly designed ideal bandpass filter with carrier frequency $f_{c}$ and bandwidth $W(\mathrm{~Hz})$ for limiting the noise power and normalizing the noise variance. Second, the output signal from the filter is squared and integrated over time duration $T$ to measure the energy of the received signal at the energy detector. The collected energy, denoted $E$, is the test statistic of the detector. For each component (in-phase or quadrature) of the received signal, the number of samples is integer $u$. Further, according to [5], the value of $u$ could be either $T W$ or $(T W+1)$, depending on the position of the first sample. In [4], it is shown that the test statistic $E$ follows a central chisquare distribution with $2 u$ degrees of freedom when the signal is absent (i.e., under hypothesis $H_{0}$ ), or follows a non-central chi-square distribution with $2 u$ degrees of freedom with the presence of the signal (i.e., under hypothesis $H_{1}$ ). Finally, the energy detector compares the test statistic $E$ with a prespecified threshold $\lambda$ and determines that the signal is present if $E>\lambda$, or absent otherwise.

The detection probability $\left(P_{d}\right)$ and false alarm probability $\left(P_{f}\right)$ are defined as the probabilities that the test statistic is larger than the threshold, given that the signal is present and absent, respectively. By using the cumulative distribution functions (CDF) of the central chi-square distribution and the non-central chi-square distribution, the two probabilities $P_{d}$ and $P_{f}$ can be calculated as [4]

$$
P_{f}(\lambda)=\operatorname{Pr}\left(E>\lambda \mid H_{0}\right)=\frac{\Gamma\left(u, \frac{\lambda}{2}\right)}{\Gamma(u)}
$$

and

$$
P_{d}(\gamma, \lambda)=\operatorname{Pr}\left(E>\lambda \mid H_{1}\right)=Q_{u}(\sqrt{2 \gamma}, \sqrt{\lambda}),
$$

respectively. Here, $Q_{u}(\cdot, \cdot)$ is the generalized Marcum-Q function, $\Gamma(\cdot, \cdot)$ is the upper incomplete gamma function defined as $\Gamma(a, x) \triangleq \int_{x}^{\infty} t^{a-1} e^{-t} d t$, and $\Gamma(a, 0)=\Gamma(a) \triangleq$ $\int_{0}^{\infty} t^{a-1} e^{-t} d t$, and $\gamma$ is the received instantaneous SNR of the target signal at the energy detector. The instantaneous SNR of the received signal through a single diversity branch is $\gamma=h^{2} E_{s} / N_{0}$.

\section{B. Fading Channel}

We assume that the channel undergoes Nakagami- $m$ fading, a distribution that is widely used to characterize the wireless channel fading [16], with $m$ being the fading parameter. Let $\gamma$ denote the instantaneous SNR at the receiver. Given the assumption of Nakagami- $m$ fading, the SNR $\gamma$ follows a gamma distribution, denoted $f_{\gamma}(x)$. For a fading channel, the average AUC can be obtained by averaging the AUC (for instantaneous SNR value $\gamma$ ) by the distribution of $\gamma$.

\section{AREA Under the ROC CURVE (AUC)}

The ROC curve is usually illustrated as $P_{d}$ versus $P_{f}$. For two energy detectors, it is difficult to compare their performance based on visual perception of their ROC curves, since the curves may cross. On the other hand, following the Area Theorem [11], we introduce the AUC, which is equal to the area covered by the ROC curve of $P_{d}$ versus $P_{f}$. As aforementioned, the AUC is a measure of the detection capability of the energy detector. Generally, as the threshold $\lambda$ in the energy detection varies from $\infty$ to 0 , the false alarm and the detection probabilities vary from value 0 to value 1 , and accordingly, the AUC varies from 0.5 to 1 .

\section{A. AUC for Instantaneous SNR Value $\gamma$}

Consider the ROC curve of $P_{d}$ versus $P_{f}$. Let $A(\gamma)$ denote the AUC which is a function of instantaneous SNR value $\gamma$. Therefore, $A(\gamma)$ can be evaluated as

$$
A(\gamma)=\int_{0}^{1} P_{d}(\gamma, \lambda) d P_{f}(\lambda)
$$


Both $P_{f}(\lambda)$ and $P_{d}(\gamma, \lambda)$ are functions of the threshold $\lambda$. Therefore, we can apply the threshold averaging method [17] when calculating the AUC. When the value of $P_{f}(\lambda)$ varies from $0 \rightarrow 1$, it is equivalent to $\lambda$ ranging from $\infty \rightarrow 0$. Therefore, Eq. (4) can be re-written as

$$
A(\gamma)=-\int_{0}^{\infty} P_{d}(\gamma, \lambda) \frac{\partial P_{f}(\lambda)}{\partial \lambda} d \lambda
$$

where $\partial P_{f}(\lambda) / \partial \lambda$ is the partial derivative ${ }^{1}$ of $P_{f}$ with respect to $\lambda$, which is given from (2) as

$$
\frac{\partial P_{f}(\lambda)}{\partial \lambda}=-\frac{\lambda^{u-1} e^{-\frac{\lambda}{2}}}{2^{u} \Gamma(u)}
$$

After the substitution of (3) and (6) into (5), and the transformation $\sqrt{\lambda}=t$, Eq. (5) can be written as

$$
A(\gamma)=\frac{1}{2^{u-1} \Gamma(u)} \int_{0}^{\infty} t^{2 u-1} e^{-\frac{t^{2}}{2}} Q_{u}(\sqrt{2 \gamma}, t) d t .
$$

Using the following identity of the Marcum-Q function

$$
Q_{u}(\beta, \alpha)=1-Q_{u}(\alpha, \beta)+e^{-\frac{\alpha^{2}+\beta^{2}}{2}} \sum_{r=1-u}^{u-1}\left(\frac{\alpha}{\beta}\right)^{r} I_{r}(\alpha \beta)
$$

where $I_{r}(\cdot)$ is the $r$ th-order modified Bessel function of the first kind, Eq. (7) can be re-written as

$$
\begin{array}{r}
A(\gamma)=\frac{1}{2^{u-1} \Gamma(u)} \int_{0}^{\infty} t^{2 u-1} e^{-\frac{t^{2}}{2}}\left(1-Q_{u}(t, \sqrt{2 \gamma})+\right. \\
\left.e^{-\frac{2 \gamma+t^{2}}{2}} \sum_{k=1-u}^{u-1}\left(\frac{t}{\sqrt{2 \gamma}}\right)^{k} I_{k}(\sqrt{2 \gamma} t)\right) d t .
\end{array}
$$

After some mathematical manipulations and with the aid of expression (26) in the Appendix, $A(\gamma)$ can be evaluated in closed-form as (see the Appendix for the detailed derivation)

$$
\begin{aligned}
A(\gamma)=1 & -\sum_{k=0}^{u-1} \frac{1}{2^{k} k !} \gamma^{k} e^{-\frac{\gamma}{2}} \\
& +\sum_{k=1-u}^{u-1} \frac{\Gamma(u+k)}{2^{u+k} \Gamma(u)} e^{-\gamma} \tilde{F}_{1}\left(u+k ; 1+k ; \frac{\gamma}{2}\right)
\end{aligned}
$$

where ${ }_{1} \tilde{F}_{1}(\cdot ; \cdot ; \cdot)$ is the regularized confluent hypergeometric function of the confluent hypergeometric function ${ }_{1} F_{1}(\cdot ; \cdot ; \cdot)$ [18]. Note that Eq. (9) gives the AUC of an energy detector for a specific value of instantaneous SNR $\gamma$. Therefore, $A(\gamma)$ is defined as unfaded AUC. The average AUC in closed-form under the AWGN channel can be found from expression (9) after replacing $\gamma$ by $\bar{\gamma}$, where $\bar{\gamma}$ is the average SNR.

\section{B. Partial AUC}

Although the AUC is a measure of the overall detection capability, it may not always unambiguously indicate when one detector is better than another. For example, when two ROC curves cross, it is possible that the AUC for the two ROC curves is the same. This situation can arise when the two associated detectors have different performance in different regions of detection threshold $\lambda$. The area of the ROC

\footnotetext{
${ }^{1}$ Note that the false alarm probability $P_{f}$ is also a function of $u$. We omit variable $u$ from expression of $P_{f}$ for simplicity of presentation.
}

curve (for $\lambda$ from 0 to $\infty$ ) only gives the overall detection performance, but cannot differentiate the two detectors in a small region of $\lambda$, say $\lambda_{1} \leq \lambda \leq \lambda_{2}$. To remedy this drawback, the partial area under the ROC curve [19] in region $\left(\lambda_{1}, \lambda_{2}\right)$ can be used to demonstrate the difference, as given by

$$
A_{\text {Par }}(\gamma)=-\int_{\lambda_{1}}^{\lambda_{2}} P_{d}(\gamma, \lambda) \frac{\partial P_{f}(\lambda)}{\partial \lambda} d \lambda
$$

Nevertheless, the partial AUC measure appears intractable for closed-form analysis. It can however be readily evaluated via numerical integration methods that are available in the mathematical software packages such as MATHEMATICA and MATLAB. For the sake of brevity, we do not further study this measure.

\section{Average AUC over NaKagami- $m$ Fading CHANNELS}

The average AUC, $\bar{A}$, over Nakagami- $m$ channels can be evaluated through averaging (9) by the SNR distribution $\left(f_{\gamma}(x)\right)$. Therefore, $\bar{A}$ can be written as

$$
\bar{A}=\int_{0}^{\infty} A(x) f_{\gamma}(x) d x .
$$

In this section, we derive closed-form average AUC expressions for no-diversity and diversity receptions, respectively, over Nakagami- $m$ distribution which is widely employed for characterizing wireless channel fading. Further, we derive the expression of the average AUC for the high SNR approximation (i.e. $\bar{\gamma} \rightarrow \infty$ ) for each case.

\section{A. No-Diversity Reception}

If the signal amplitude follows a Nakagami- $m$ distribution, then the SNR has a probability density function (PDF) given by [20]

$$
f_{\gamma^{N a k}}(x)=\frac{1}{\Gamma(m)}\left(\frac{m}{\bar{\gamma}}\right)^{m} x^{m-1} e^{-\frac{m}{\bar{\gamma}} x}, x \geq 0
$$

where $\bar{\gamma}$ is the average SNR and $m$ is Nakagami fading parameter. The average AUC for Nakagami- $m$ fading channel with no diversity, $\bar{A}_{N a k}$, can be evaluated through averaging $A(\gamma)$ in (9) by the SNR distribution $f_{\gamma^{N a k}}(x)$ given in (12). $\bar{A}_{N a k}$ can be written for integer $m$ in closed-form as (see the Appendix for the detailed derivation) in (13) on the next page, where ${ }_{2} \tilde{F}_{1}(\cdot ; \cdot ; \cdot ; \cdot)$ is the regularized confluent hypergeometric function of the confluent hypergeometric function ${ }_{2} F_{1}(\cdot ; \cdot ; \cdot)$ [18]. When $m=1$, the result in (13) means the average AUC over a Rayleigh fading channel.

For higher $\bar{\gamma}, \bar{A}_{N a k}$ in (13) can be approximated as in (14) on the next page, where $g^{N a k}(m, u)$ is the term in the square brackets which depends on parameters $m$ and $u$. When $m$ increases, the average AUC converges to 1 , and the convergence speed is with the order of $m$. So we define $m$ as the detection diversity gain or detection diversity order. 


$$
\bar{A}_{N a k}=1-\frac{1}{\Gamma(m)}\left(\frac{2 m}{2 m+\bar{\gamma}}\right)^{m} \sum_{k=0}^{u-1} \frac{\Gamma(k+m)}{k !}\left(\frac{\bar{\gamma}}{2 m+\bar{\gamma}}\right)^{k}+\left(\frac{m}{m+\bar{\gamma}}\right)^{m} \sum_{k=1-u}^{u-1} \frac{\Gamma(u+k)}{2^{u+k} \Gamma(u)} \tilde{F}_{1}\left(m ; u+k ; 1+k ; \frac{\bar{\gamma}}{2(m+\bar{\gamma})}\right)
$$

$$
\begin{aligned}
\bar{A}_{N a k} & \approx 1-\left[\frac{(2 m)^{m}}{\Gamma(m)} \sum_{k=0}^{u-1} \frac{\Gamma(k+m)}{k !}-\sum_{k=1-u}^{u-1} \frac{m^{m} \Gamma(u+k)}{2^{u+k} \Gamma(u)}{ }_{2} \tilde{F}_{1}\left(m ; u+k ; 1+k ; \frac{1}{2}\right)\right] \bar{\gamma}^{-m} \\
& =1-g^{N a k}(m, u) \bar{\gamma}^{-m} .
\end{aligned}
$$

\section{B. Diversity Reception}

Diversity combining techniques are used at the receiver to increase the receiver SNR. Next we derive the average AUC under MRC, SLC and SC diversity receptions. The $L$ diversity paths are independent and identically distributed (i.i.d.) over Nakagami- $m$ fading channels. And $\gamma_{k}$ is the SNR in the $k$ th branch.

1) Maximal Ratio Combining (MRC): MRC is a coherent combining method, which requires channel estimations. Thus the use of MRC with energy detection is not desirable. In this case, the energy detector measures the energy of the MRC combined signal rather than measuring the energy of each individual branch before combining (e.g. in square-law combining). Nevertheless, the use of MRC with energy detection deserves investigation. For instance, the ROC analysis of the energy detector with MRC reception has been studied in [4], [21]-[23]. The performance of this setup serves as an upper bound of the achievable performance by a combination of energy detection and any other diversity scheme.

In MRC, all the diversity branches are coherently combined, and the instantaneous SNR at the output of the combiner is $\gamma^{M R C}=\sum_{k=1}^{L} \gamma_{k}$. The PDF of $\gamma^{M R C}$ for i.i.d. Nakagami- $m$ fading channels is given by [20]

$$
f_{\gamma^{M R C}}(x)=\frac{1}{\Gamma(L m)}\left(\frac{m}{\bar{\gamma}}\right)^{L m} x^{L m-1} e^{-\left(\frac{m}{\bar{\gamma}}\right) x}, \quad x \geq 0 .
$$

Similar to the derivation of (13) in the Appendix, the average AUC under MRC, $\bar{A}_{N a k}^{M R C}$, can be evaluated through averaging $A(\gamma)$ in (9) by the SNR distribution in (15), given as in (16) on the next page. For higher $\bar{\gamma}, \bar{A}_{N a k}^{M R C}$ in (16) can be approximated as in (17) on the next page, where $g^{M R C}(m, L, u)$ is the term in the square brackets which depends on parameters $m, L$ and $u$. The detection diversity gain is equal to $L m$.

2) Square-Law Combining (SLC): In contrast to the MRC, $L$ diversity branches in SLC are combined after the received signal from each branch is squared and integrated (over period $T)$. The energy detector receives the sum of $L$ decision statistics. Therefore, the resultant decision statistic follows a central chi-square distribution with $2 L u$ degrees of freedom and a non-central chi-square distribution with $2 \mathrm{Lu}$ degrees of freedom under hypothesis $H_{0}$ and $H_{1}$, respectively. The non-centrality parameter under hypothesis $H_{1}$ is $\gamma^{S L C}=$ $\sum_{k=1}^{L} \gamma_{k}$. The false alarm and the detection probabilities $P_{f}(\lambda)$ and $P_{d}\left(\gamma^{S L C}, \lambda\right)$ under AWGN channel are shown to be given by (2) and (3) with $u$ and $\gamma$ being replaced by $L u$ and $\gamma^{S L C}$, respectively [5].
It can be seen that the AUC under SLC for AWGN channel is equivalent to $A(\gamma)$ in (9), after replacing $u$ by $L u$. Since $\gamma^{S L C}$ and $\gamma^{M R C}$ have similar expression $\left(\sum_{k=1}^{L} \gamma_{k}\right)$, the average AUC under SLC with Nakagami- $m$ fading channels, $\bar{A}_{N a k}^{S L C}$, can be evaluated as $\bar{A}_{N a k}^{M R C}$ in (16) after replacing $u$ by $L u$. Further, high average SNR approximation can be derived as

$$
\bar{A}_{N a k}^{S L C} \approx 1-g^{S L C}(m, L, u) \bar{\gamma}^{-L m}
$$

where $g^{S L C}(m, L, u)$ is equivalent to $g^{M R C}(m, L, u)$ after replacing $u$ by $L u$. The detection diversity gain is equal to $\mathrm{Lm}$.

3) Selection Combining (SC): In SC, the branch with the strongest SNR among all diversity branches is selected. The instantaneous SNR at the output of the combiner is $\gamma^{S C}=\max \left\{\gamma_{1}, \ldots, \gamma_{L}\right\}$. The PDF of $\gamma^{S C}$ for $i . i . d$. Nakagami$m$ fading channels with integer $m$ is given by [24]

$$
\begin{aligned}
f_{\gamma^{S C}}(x)= & \frac{L}{\Gamma(m)} \sum_{l=0}^{L-1}(-1)^{l}\left(\begin{array}{c}
L-1 \\
l
\end{array}\right) e^{-\frac{(l+1) m}{\bar{\gamma}} x} \\
& \sum_{n=0}^{l(m-1)} \beta(n, l, m)\left(\frac{m}{\bar{\gamma}}\right)^{m+n} x^{m+n-1}, x \geq 0
\end{aligned}
$$

where $\beta(n, l, m)$ is the notation defined in [24]. Similar to the derivation of (13) in the Appendix, average AUC under SC with Nakagami- $m$ fading channels, $\bar{A}_{N a k}^{S C}$, can be evaluated as in (20) on the next page. For higher $\bar{\gamma}, \bar{A}_{N a k}^{S C}$ in (20) can be approximated as in (21) on the next page, where $g^{S C}(m, L, l, u, n)$ is the term in the square brackets. The effective detection diversity gain is equal to $\mathrm{Lm}$.

\section{Average AUC of MRC Under Rayleigh Fading WITH CHANNEL ESTIMATION ERRORS AND CHANNEL FADING CORRELATIONS}

\section{A. Impact of Channel Estimation Errors}

In Section IV, the average AUC of MRC is derived by assuming that each branch is weighted with its perfect channel knowledge (i.e. perfect channel estimation). But in practice, channel estimation errors are inevitable. So it is important to incorporate the effect of channel estimation errors on the average AUC. Assuming that the complex Gaussian error is accumulated to each weighting factor in the combiner, a PDF of the output SNR is derived in [25] for MRC under Rayleigh fading channels. By re-arranging the terms of the results in [25], an alternative form of the PDF of the output SNR of 


$$
\begin{aligned}
\bar{A}_{N a k}^{M R C}=1 & -\frac{1}{\Gamma(L m)}\left(\frac{2 m}{2 m+\bar{\gamma}}\right)^{L m} \sum_{k=0}^{u-1} \frac{\Gamma(k+m)}{k !}\left(\frac{\bar{\gamma}}{2 m+\bar{\gamma}}\right)^{k} \\
& +\left(\frac{m}{m+\bar{\gamma}}\right)^{L m} \sum_{k=1-u}^{u-1} \frac{\Gamma(u+k)}{2^{u+k} \Gamma(u)} \tilde{F}_{1}\left(L m, u+k ; 1+k ; \frac{\bar{\gamma}}{2(m+\bar{\gamma})}\right)
\end{aligned}
$$

$$
\begin{aligned}
\bar{A}_{N a k}^{M R C} & \approx 1-\left[\frac{(2 m)^{L m}}{\Gamma(L m)} \sum_{k=0}^{u-1} \frac{\Gamma(k+m)}{k !}-\sum_{k=1-u}^{u-1} \frac{m^{L m} \Gamma(u+k)}{2^{u+k} \Gamma(u)}{ }_{2} \tilde{F}_{1}\left(L m ; u+k ; 1+k ; \frac{1}{2}\right)\right] \bar{\gamma}^{-L m} \\
& =1-g^{M R C}(m, L, u) \bar{\gamma}^{-L m} .
\end{aligned}
$$

$$
\begin{aligned}
\bar{A}_{N a k}^{S C}= & 1-\sum_{l=0}^{L-1} \frac{(-1)^{l} L \beta(n, l, m)}{\Gamma(m)}\left(\begin{array}{c}
L-1 \\
l
\end{array}\right)\left(\frac{m}{\bar{\gamma}}\right)^{m+n}\left[\sum_{k=0}^{u-1} \frac{\Gamma(k+m+n)}{2^{k} k !}\left(\frac{2 \bar{\gamma}}{2 m(l+1)+\bar{\gamma}}\right)^{k+m+n}\right. \\
& \left.-\sum_{k=1-u}^{u-1} \frac{\Gamma(u+k) \Gamma(m+n)}{2^{u+k} \Gamma(u)}\left(\frac{\bar{\gamma}}{\bar{\gamma}+m(l+1)}\right)^{m+n}{ }_{2} \tilde{F}_{1}\left(m+n ; u+k ; 1+k ; \frac{\bar{\gamma}}{2(m(l+1)+\bar{\gamma})}\right)\right] .
\end{aligned}
$$

$$
\begin{aligned}
\bar{A}_{N a k}^{S C} \approx & 1-\sum_{l=0}^{L-1} \sum_{n=0}^{l(m-1)}\left[\frac { ( - 1 ) ^ { l } L \beta ( n , l , m ) m ^ { m + n } } { \Gamma ( m ) } ( \begin{array} { c } 
{ L - 1 } \\
{ l }
\end{array} ) \left(\sum_{k=0}^{u-1} \frac{2^{m+n} \Gamma(k+m+n)}{k !}\right.\right. \\
& \left.\left.-\sum_{k=1-u}^{u-1} \frac{\Gamma(u+k) \Gamma(m+n)}{2^{u+k} \Gamma(u)}{ }_{2} \tilde{F}_{1}\left(m+n ; u+k ; 1+k ; \frac{1}{2}\right)\right)\right] \bar{\gamma}^{-(m+n)} \\
= & 1-\sum_{l=0}^{L-1} \sum_{n=0}^{l(m-1)} g^{S C}(m, L, l, u, n) \bar{\gamma}^{-(m+n)}
\end{aligned}
$$

MRC under $i . i . d$. Rayleigh fading channels, $f_{\gamma^{E r r}}(x)$, is given in [26]. Applying the definition of Bernstein polynomials, $B_{i}^{n}(t)=\left(\begin{array}{l}n \\ i\end{array}\right) t^{i}(1-t)^{n-i}$ [27], the PDF $f_{\gamma^{E r r}}(x)$ can be rewritten as

$$
f_{\gamma^{E r r}}(x)=\sum_{l=1}^{L} B_{l-1}^{L-1}\left(\rho^{2}\right) \frac{1}{(l-1) ! \bar{\gamma}^{l}} x^{l-1} e^{-\frac{x}{\gamma}}, \quad x \geq 0
$$

where $\rho$ is the correlation coefficient between the correct complex channel gain and the estimated complex channel gain $(0 \leq \rho \leq 1)$. So $\rho$ represents the channel estimation accuracy level. The average AUC in this case, $\bar{A}_{E r r}$, can be evaluated through averaging $A(\gamma)$ in (9) by the SNR distribution $f_{\gamma E r r}(x)$ given in (22). Similar to the derivation of (13) in the Appendix, $\bar{A}_{E r r}$ can be evaluated as in (23) on the next page.

\section{B. Impact of Channel Fading Correlations}

In the previous sections, we assume that the diversity branches are independent with each other. However, in practice, this assumption is not always valid. Therefore, it is important to analyze the performance of the energy detector with correlated fading channels. Since there are multiple correlation scenarios, we do not have space to consider all interesting cases. Instead, we consider one simple yet instructive case: a dual-branch MRC receiver under correlated and identically distributed Rayleigh fading. The PDF of output SNR, $\gamma^{C r r}$, is given as [28]

$$
f_{\gamma \text { Crr }}(x)=\frac{1}{2 \sqrt{\mu} \bar{\gamma}}\left(e^{-\frac{x}{(1+\sqrt{\mu}) \bar{\gamma}}}-e^{-\frac{x}{(1-\sqrt{\mu}) \bar{\gamma}}}\right), \quad x \geq 0
$$

where $\mu$ is the power correlation coefficient of dual-branch signals $(0<\mu \leq 1)$. The average AUC, $\bar{A}_{C r r}$, can be evaluated through averaging $A(\gamma)$ in (9) by the SNR distribution $f_{\gamma \mathrm{Crr}}(x)$ in (24), which is given in (25) on the next page with $a_{1}=1 /(1+\sqrt{\mu}) \bar{\gamma}$ and $a_{2}=1 /(1-\sqrt{\mu}) \bar{\gamma}$.

\section{Numerical and Simulation Results}

In this section, we present numerical and Monte-Carlo simulation results. Since the average AUC depends on parameters such as $m, u, \bar{\gamma}$ and $L$ (if diversity reception is used), several different cases are discussed here. Analytical expressions in (13), (16), (20), (23) and (25) for average AUCs are verified by numerical calculations and by Monte-Carlo simulations using MATHEMATICA and MATLAB software packages, respectively. Continuous and dashed lines in the following figures represent numerical values, while discrete signs represent simulation values.

Fig. 1 shows the analytical and simulation results for average AUC with no diversity reception under Nakagami$m$ fading model. The analytical results are based on (13). 


$$
\begin{aligned}
\bar{A}_{E r r}= & 1-\sum_{l=1}^{L} B_{l-1}^{L-1}\left(\rho^{2}\right) \frac{1}{(l-1) ! \bar{\gamma}^{l}}\left[\sum_{k=0}^{u-1} \frac{2^{l} \Gamma(k+l)}{k !}\left(\frac{\bar{\gamma}}{\bar{\gamma}+2}\right)^{k+l}\right. \\
& \left.-\sum_{k=1-u}^{u-1} \frac{\Gamma(u+k) \Gamma(l)}{2^{u+k} \Gamma(u)}\left(\frac{\bar{\gamma}}{1+\bar{\gamma}}\right)^{l}{ }_{2} \tilde{F}_{1}\left(l ; u+k ; 1+k ; \frac{\bar{\gamma}}{2(1+\bar{\gamma})}\right)\right] .
\end{aligned}
$$

$$
\begin{aligned}
\bar{A}_{C r r}=1-\frac{1}{2 \sqrt{\mu} \bar{\gamma}} & {\left[\sum_{k=0}^{u-1} \frac{\Gamma(k+l)}{2^{k} k !}\left(\frac{1}{\left(a_{1}+\frac{1}{2}\right)^{k+1}}-\frac{1}{\left(a_{2}+\frac{1}{2}\right)^{k+1}}\right)-\sum_{k=1-u}^{u-1} \frac{\Gamma(u+k)}{2^{u+k} \Gamma(u)}\right.} \\
& \left.\left(\frac{{ }_{2} \tilde{F}_{1}\left(1 ; u+k ; 1+k ; \frac{1}{2\left(1+a_{1}\right)}\right)}{1+a_{1}}-\frac{\tilde{F}_{1}\left(1 ; u+k ; 1+k ; \frac{1}{2\left(1+a_{2}\right)}\right)}{1+a_{2}}\right)\right] .
\end{aligned}
$$

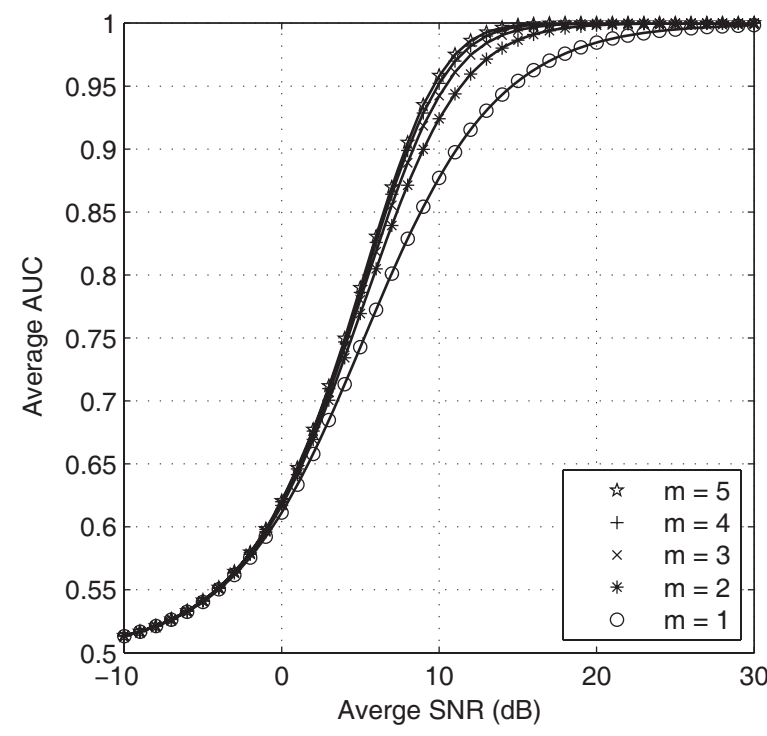

Fig. 1. Average AUC versus average SNR $\bar{\gamma}$ with no diversity case $(u=4)$.

This figure shows the average AUC versus average SNR with different fading parameter $m$ values. Note that numerical and simulation results match well. Note also that a higher $m$ leads to larger average AUC, and thus, higher overall detection capability. This is because the average AUC converges to unity faster when the average SNR and the fading index $m$ increase, as the detection diversity order is equal to $m$ based on (14). Since the accuracy of expression (13) is verified in Fig. 1 by comparison of numerical and simulation results, to avoid clutter, simulation results are not shown in Fig. 2, Fig. 3 and Fig. 4.

Also for the no-diversity case, Fig. 2 shows (by solid lines) the average AUC versus fading parameter $m$ with different average SNR values. It can be seen that, between the average SNR and the fading parameter, the average SNR is the dominant factor in determining the detection capability, particularly in the low-SNR region. The average AUC reaches unity even for the small values of $m$ when the average SNR is high (e.g. $\bar{\gamma}>15 \mathrm{~dB}$ ), as explained in (14). For higher $m$ values, there is an asymptotic value of the average AUC for a specific average SNR value. When $m \rightarrow \infty$, the fading

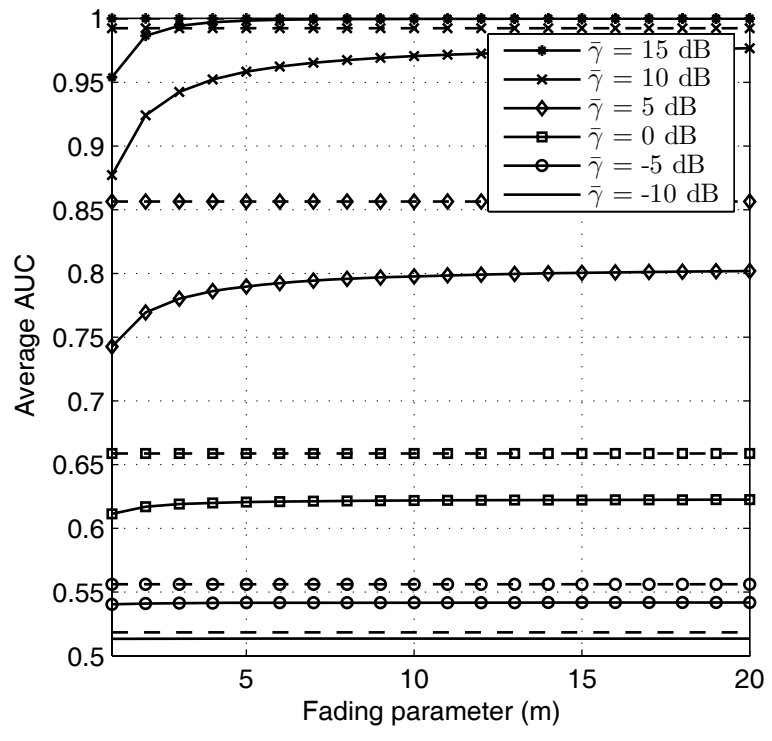

Fig. 2. The Average AUC as a function of fading parameter $m(u=4)$.

channel is equivalent to the AWGN channel. The average AUC under the AWGN channel is equivalent to expression (9) after replacing $\gamma$ by $\bar{\gamma}$, which is also plotted in Fig. 2 (by dashed lines) as the asymptotic value of the average AUC of the Nakagami- $m$ fading channel.

The influence of the number of samples $u$ on the AUC performance is investigated in Figs. 3 and 4. When the fading parameter is fixed at $m=2$, Fig. 3 shows the average AUC versus the average SNR for different $u$ values, while Fig. 4 shows the average AUC versus $u$ with different average SNR values. Somewhat paradoxically, a higher number of samples $u$ tends towards a lower detection capability. The reason is as follows. When the value of $u$ increases, the detection and false alarm probabilities both increase. However, the false alarm probability increases faster than the detection probability, thus leading to a lower overall detection capability. Nevertheless, in the high SNR region $(\bar{\gamma}>15 \mathrm{~dB})$, the differences among different AUC values for different $u$ values peter out.

For diversity reception case, when $u$ is fixed at $u=4$ and fading parameter is fixed at $m=2$, Fig. 5 shows the analytical and simulation results for the average AUC as a function 


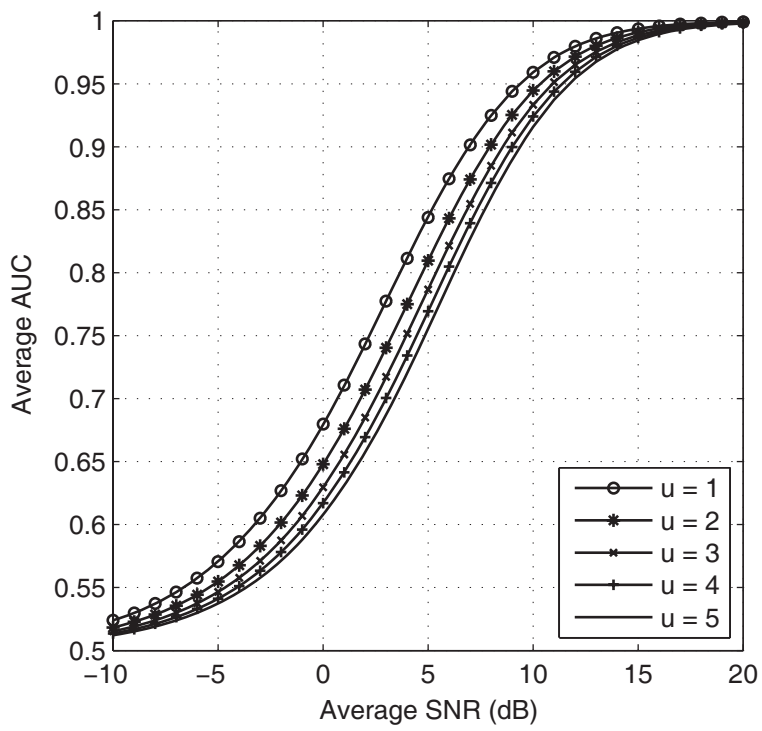

Fig. 3. Average AUC versus average SNR for different $u$ with no diversity $(m=2)$.

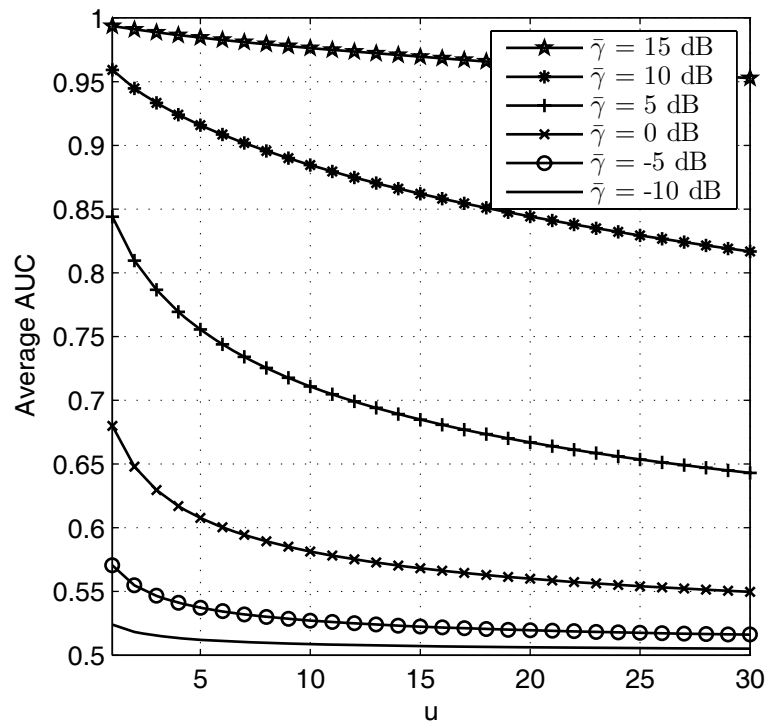

Fig. 4. Average AUC versus the number $u$ of samples for different average SNR $\bar{\gamma}$ with no diversity $(m=2)$.

of the average SNR for different values of diversity branch number $L$, while Fig. 6 shows the analytical and simulation results for the average AUC as a function of the number of branches $L$ for different average SNR. As can be expected, Figs. 5 and 6 show that MRC always outperforms SLC and SC. For a specific AUC value, say 0.75, the MRC scheme with five diversity branches gains about $8 \mathrm{~dB}$ in terms of the average SNR. Moreover, it can be seen that, with the increase of $L$, the average AUC in MRC and SLC approaches unity much faster than the average AUC in SC. The reason is the difference of the SNR after the combiners, for which we have $\gamma^{M R C}>\gamma^{S L C}>\gamma^{S C}$. Note that the detection diversity gains of the three combiners are all $\mathrm{Lm}$. A similar observation is also found in [4] for dual-branch MRC and SC.

Note that the cost of using MRC is the requirement of high-quality channel estimates. The performance of MRC is

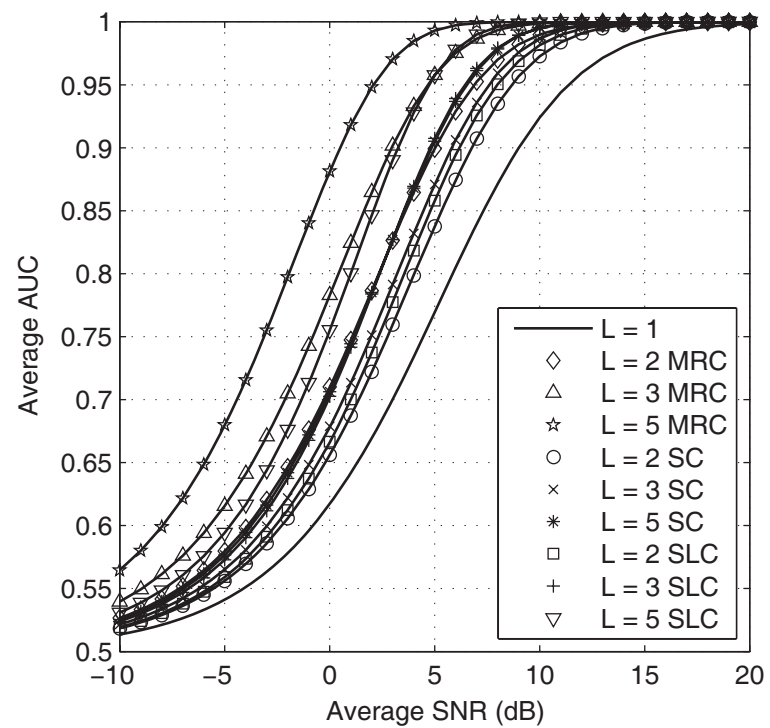

Fig. 5. Average AUC versus average SNR with different $L$ in diversity receptions $(u=4$ and $m=2)$.

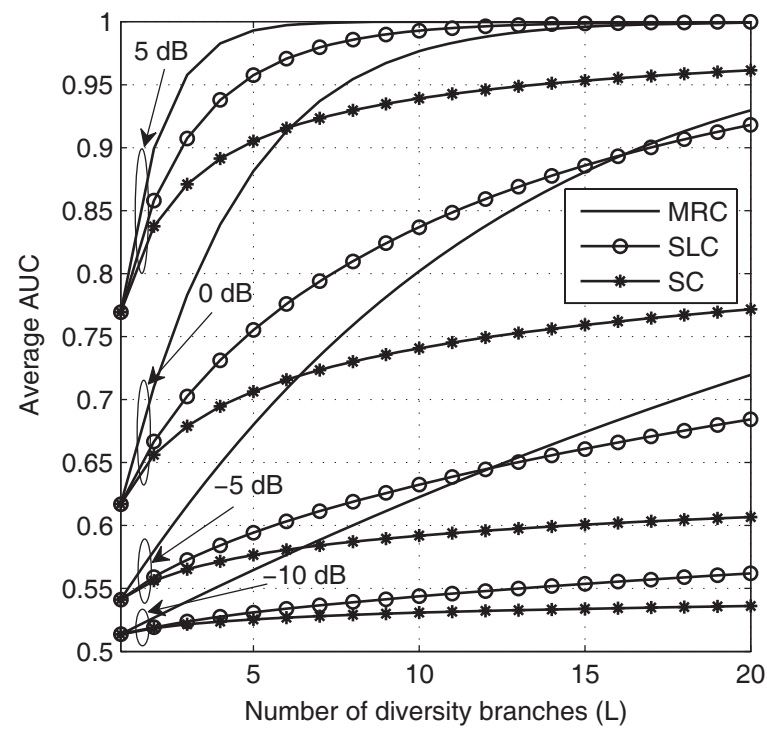

Fig. 6. Average AUC versus branch number for different average SNR in diversity receptions $(u=4$ and $m=2)$.

likely to deteriorate with poor channel quality estimates. Since high-quality estimates are unlikely to be present for energydetection-type applications, an interesting question is how good the quality of channel estimates should be in order for MRC to outperform SLC (which does not require channel estimates). This question is answered in Fig. 7, which shows the analytical and simulation results for the average AUC (for MRC) versus the average SNR with different level of channel estimation accuracy $(\rho)$. It can be seen that the simulation and analytical results match well. The average AUC degrades as $\rho$ changes from 1 (perfect channel estimation) to 0.5. As a comparison, the performance with SLC is also presented in Fig. 7 by a dashed line. In this particular example, we can see that if $\rho$ is less than 0.6 , it is better to implement SLC receiver rather than implementing a post-detection MRC receiver.

Fig. 8 shows the average AUC versus the average SNR 


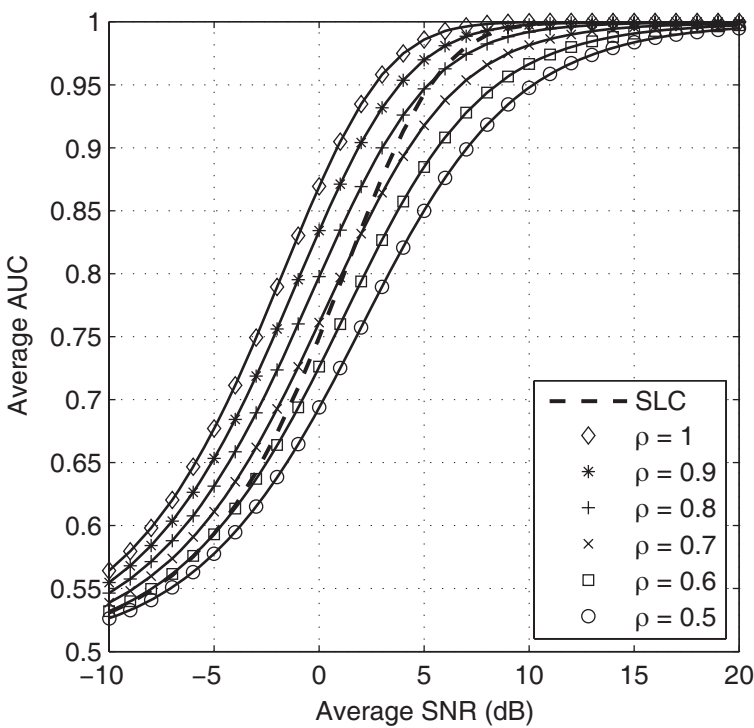

Fig. 7. Average AUC versus average SNR for MRC with different $\rho(u=4$ and $m=1$ ).

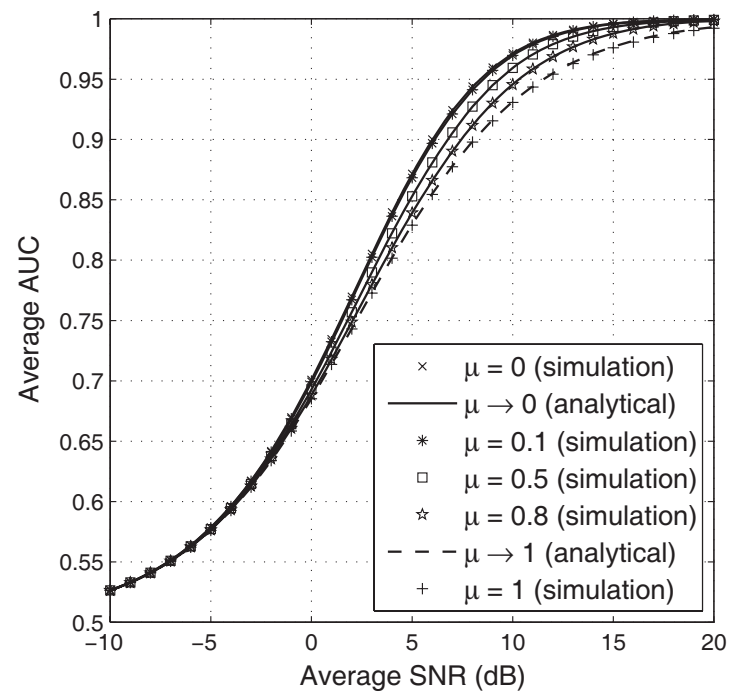

Fig. 8. Average AUC versus average SNR for dual-branch MRC with different $\mu(u=4$ and $m=1)$.

with different channel correlation coefficient $(\mu)$ for dualbranch MRC. The average AUC is seen to degrade as the correlation between the two branches increases. If channels are fully correlated $(\mu=1)$ and fully uncorrelated $(\mu=0)$, it corresponds to the single-branch case and the independentdual-branch MRC case, respectively. Since expression (25) is valid for $0<\mu<1$, in Fig. 8 we plot numerically calculated average AUC for $\mu \rightarrow 1$ and $\mu \rightarrow 0$. Both curves match well with fully correlated $(\mu=1)$ and fully uncorrelated $(\mu=0)$ Monte-Carlo simulation results, respectively.

\section{CONCLUSIONS}

A simple figure of merit characterizing the performance of an energy detector is the AUC. The AUC is simply the area under the ROC curve. No AUC results appear to be available in the wireless literature. In this paper, the average AUC of an energy detector is derived for Nakagami- $m$ fading channels with no-diversity based on threshold averaging technique. The average AUC derivation is then extended to diversity reception cases such as selection combining, square-law combining, and maximal ratio combining with Nakagami- $m$ fading diversity branches. The impacts of channel estimation errors and channel fading correlations are also investigated. High SNR approximations and detection diversity gain are also derived. We anticipate that the AUC measure will also be useful for characterizing the performance of other numerous detection algorithms.

\section{APPENDIX}

\section{A. Necessary Integrations}

Some integrations necessary for the rest of the derivations are presented below.

First, we define

$$
J_{1}(a, p, r, c) \triangleq \int_{0}^{\infty} x^{a-1} e^{-p x^{2}} I_{r}(c x) d x
$$

with parameters $a, p, r, c$, where $\operatorname{Re}[a]>0 \wedge \operatorname{Re}[p]>0^{2}$. Although a closed-form solution for $J_{1}(a, p, r, c)$ is available in [29, eq. (2.15.5.4)], it can not be applied for negative integer values of $r$. Therefore, we present an alternative method for any integer $r$, as follows.

After applying series expansion of $I_{r}(c x)$ in (26), and with transformation $t=x^{2}, J_{1}(a, p, r, c)$ can be written as

$$
J_{1}(a, p, r, c)=\sum_{k=0}^{\infty} \frac{\left(\frac{c}{2}\right)^{2 k+r}}{\Gamma(k+r+1) k !} \frac{1}{2} \int_{0}^{\infty} t^{k+\frac{r+a}{2}-1} e^{-p t} d t .
$$

Further, $J_{1}(a, p, r, c)$ can be shown to be

$$
\begin{aligned}
J_{1}(a, p, r, c)= & \frac{c^{r}}{2^{r+1}} p^{-\frac{a+r}{2}} \Gamma\left(\frac{a+r}{2}\right) \\
& \sum_{k=0}^{\infty} \frac{\left(\frac{a+r}{2}\right)_{k} p^{-k}}{\Gamma(k+r+1) k !}\left(\frac{c^{2}}{4}\right)^{k}
\end{aligned}
$$

where $(n)_{k}$ is the Pochhammer symbol defined as $(n)_{k}=$ $\frac{\Gamma(n+k)}{\Gamma(n)}[30]$.

Given a hypergeometric or generalized hypergeometric function ${ }_{p} F_{q}\left(a_{1}, \ldots, a_{p} ; b_{1}, \ldots, b_{q} ; z\right)$, the corresponding regularized hypergeometric function is defined as [18, eq. 07.32.02.0001.01]

$$
\begin{aligned}
{ }_{p} \tilde{F}_{q}\left(a_{1}, \ldots, a_{p} ; b_{1}, \ldots, b_{q} ; z\right) & \triangleq \frac{{ }_{p} F_{q}\left(a_{1}, \ldots, a_{p} ; b_{1}, \ldots, b_{q} ; z\right)}{\Gamma\left(b_{1}\right) \ldots \Gamma\left(b_{q}\right)} \\
& =\sum_{k=0}^{\infty} \frac{\prod_{j=1}^{p}\left(a_{j}\right)_{k} z^{k}}{k ! \prod_{j=1}^{q} \Gamma\left(k+b_{j}\right)} .
\end{aligned}
$$

Therefore, $J_{1}(a, p, r, c)$ can be evaluated as

$$
J_{1}(a, p, r, c)=\frac{c^{r} p^{-\frac{a+r}{2}} \Gamma\left(\frac{a+r}{2}\right)}{2^{r+1}}{ }_{1} \tilde{F}_{1}\left(\frac{a+r}{2} ; r+1 ; \frac{c^{2}}{4 p}\right) .
$$

Next, we define

$$
J_{2}(a, p, b, d, c) \triangleq \int_{0}^{\infty} x^{a-1} e^{-p x}{ }_{1} \tilde{F}_{1}(b ; d ; c x) d x
$$

\footnotetext{
${ }^{2}$ Here $\wedge$ stands for AND.
} 
with parameters $a, p, b, d, c$, where $\operatorname{Re}[a]>0 \wedge \operatorname{Re}[p]>0$. Using the definition of the regularized hypergeometric functions ${ }_{p} \tilde{F}_{q}$ in (27), $J_{2}(a, p, b, d, c)$ can be solved as

$$
\begin{aligned}
J_{2}(a, p, b, d, c) & =\sum_{k=0}^{\infty} \frac{(a)_{k} c^{k}}{k ! \Gamma(b+k)} \int_{0}^{\infty} x^{k+a-1} e^{-p x} d x \\
& =\sum_{k=0}^{\infty} \frac{(a)_{k} c^{k}}{k ! \Gamma(b+k)} \frac{\Gamma(k+a)}{p^{k+a}} .
\end{aligned}
$$

With the relationship of $\Gamma(k+a)=(a)_{k} \Gamma(a)$ for integer values of $a, J_{2}(a, p, b, d, c)$ is shown to be

$$
J_{2}(a, p, b, d, c)=p^{-a} \Gamma(a)_{2} \tilde{F}_{1}\left(a ; b ; d ; \frac{c}{p}\right) .
$$

\section{B. Derivation of $A(\gamma)$ in (9)}

Eq. (8) can be written as

$$
A(\gamma)=\frac{1}{2^{u-1} \Gamma(u)}\left(I_{1}-I_{2}+I_{3}\right)
$$

where $I_{1}, I_{2}$ and $I_{3}$ are defined as follows

$$
\begin{gathered}
I_{1} \triangleq \int_{0}^{\infty} t^{2 u-1} e^{-\frac{t^{2}}{2}} d t=2^{2 u-1} \Gamma(u) \\
I_{2} \triangleq \int_{0}^{\infty} t^{2 u-1} e^{-\frac{t^{2}}{2}} Q_{u}(t, \sqrt{2 \gamma}) d t \\
=2^{2 u-1}(u-1) ! e^{-\frac{\gamma}{2}} \sum_{k=0}^{u-1} \frac{1}{k !}\left(\frac{\gamma}{2}\right)^{k} \\
I_{3} \triangleq e^{-\gamma} \sum_{k=1-u}^{u-1}\left(\frac{1}{2 \gamma}\right)^{\frac{k}{2}} \int_{0}^{\infty} t^{2 u+k-1} e^{-t^{2}} I_{k}(\sqrt{2 \gamma} t) d t \\
=e^{-\gamma} \sum_{k=1-u}^{u-1} 2^{-(1+k)} \Gamma(u+k){ }_{1} \tilde{F}_{1}\left(u+k ; 1+k ; \frac{\gamma}{2}\right)
\end{gathered}
$$

where the second equality of $I_{2}$ is from [31, eq. (28)], and the second equality of $I_{3}$ is from (26) and $(28) .{ }_{1} \tilde{F}_{1}(\cdot ; \cdot ; \cdot)$ is the regularized confluent hypergeometric function of ${ }_{1} F_{1}$ [18].

After replacing $I_{1}, I_{2}$, and $I_{3}$ by the above definitions, Eq. (31) is exactly (9).

\section{Derivation of $\bar{A}_{N a k}$ in (13)}

With (9), (11), (12) and the fact $\int_{0}^{\infty} f_{\gamma}(x)=1, \bar{A}_{N a k}$ can be written as

$$
\begin{aligned}
\bar{A}_{N a k}=1 & -\frac{1}{\Gamma(m)}\left(\frac{m}{\bar{\gamma}}\right)^{m} \sum_{k=0}^{u-1} \frac{1}{2^{k} k !} I_{4} \\
& +\frac{1}{\Gamma(m)}\left(\frac{m}{\bar{\gamma}}\right)^{m} \sum_{k=1-u}^{u-1} \frac{\Gamma(u+k)}{2^{u+k} \Gamma(u)} I_{5}
\end{aligned}
$$

where $I_{4}$ and $I_{5}$ are defined as

$$
\begin{aligned}
I_{4} & \triangleq \int_{0}^{\infty} x^{m+k-1} e^{-\left(\frac{m}{\bar{\gamma}}+\frac{1}{2}\right) x} d x \\
& =\Gamma(k+m)\left(\frac{m}{\bar{\gamma}}+\frac{1}{2}\right)^{-(k+m)}
\end{aligned}
$$

and

$$
I_{5} \triangleq \int_{0}^{\infty} x^{m-1} e^{-\left(\frac{m}{\bar{\gamma}}+1\right) x}{ }_{1} \tilde{F}_{1}\left(u+k ; 1+k ; \frac{x}{2}\right) d x .
$$

Using the transformation $x=2 y$ and based on (29) and (30), $I_{5}$ can be evaluated for integer $m$ as

$$
I_{5}=\frac{\Gamma(m)}{\left(\frac{m}{\bar{\gamma}}+1\right)^{m}}{ }_{2} \tilde{F}_{1}\left(m ; u+k ; 1+k ; \frac{\bar{\gamma}}{2(m+\bar{\gamma})}\right) \text {. }
$$

After replacing $I_{4}$ by (33) and replacing $I_{5}$ by (34), Eq. (32) is exactly Eq. (13).

\section{ACKNOWLEDGMENTS}

The authors would like to thank the Editor Dr. G. Colavolpe and the anonymous reviewers for their constructive comments which improve the presentation of this paper.

\section{REFERENCES}

[1] J. P. Egan, Signal Detection Theory and ROC Analysis. New York: Academic Press, 1975.

[2] H. Urkowitz, "Energy detection of unknown deterministic signals," Proc. IEEE, vol. 55, no. 4, pp. 523-531, Apr. 1967.

[3] V. I. Kostylev, "Energy detection of a signal with random amplitude," in Proc. IEEE Int. Conf. Commun. (ICC), Apr. 2002, pp. 1606-1610.

[4] F. F. Digham, M. S. Alouini, and M. K. Simon, "On the energy detection of unknown signals over fading channels," in Proc. IEEE Int. Conf. Commun. (ICC), May 2003, pp. 3575-3579.

[5] F. F. Digham, M. S. Alouini, and M. K. Simon, "On the energy detection of unknown signals over fading channels," IEEE Trans. Commun., vol. 55, no. 1, pp. 21-24, Jan. 2007.

[6] S. P. Herath and N. Rajatheva, "Analysis of equal gain combining in energy detection for cognitive radio over Nakagami channels," in Proc. IEEE Global Commun. Conf. (GLOBECOM), Nov.-Dec. 2008.

[7] S. Atapattu, C. Tellambura, and H. Jiang, "Relay based cooperative spectrum sensing in cognitive radio networks," in Proc. IEEE Global Commun. Conf. (GLOBECOM), Nov.-Dec. 2009.

[8] S. Atapattu, C. Tellambura, and H. Jiang, "Performance of an energy detector over channels with both multipath fading and shadowing," IEEE Trans. Wireless Commun., submitted.

[9] J. A. Swets, R. M. Dawes, and J. Monahan, "Better decisions through science," Scientific American, vol. 283, no. 4, pp. 82-87, Oct. 2000.

[10] F. J. Provost and T. Fawcett, "Robust classification for imprecise environments," Machine Learning, vol. 42, no. 3, pp. 203-231, Mar. 2001.

[11] T. D. Wickens, Elementary Signal Detection Theory. New York: Oxford University Press, 2002

[12] J. A. Hanley and B. J. Mcneil, "The meaning and use of the area under a receiver operating characteristic (ROC) curve," Radiology, vol. 143, no. 1, pp. 29-36, Apr. 1982.

[13] J. H. Shapiro, "Bounds on the area under the ROC curve," J. Opt. Soc. Am. A, vol. 16, no. 1, pp. 1963-1968, Jan. 1999.

[14] E. Clarkson, "Bounds on the area under the receiver operating characteristic curve for the ideal observer," J. Opt. Soc. Am. A, vol. 19, no. 10, pp. 1963-1968, Oct. 2002.

[15] H. H. Barrett, C. K. Abbey, and E. Clarkson, "Objective assessment of image quality-III: ROC metrics, ideal observers, and likelihoodgenerating functions," J. Opt. Soc. Am. A, vol. 15, no. 6, pp. 1520-1535, June 1998.

[16] M. Nakagami, "The $m$-distribution, a general formula for intensity distribution of rapid fading," in Statistical Methods in Radio Wave Propagation, W. G. Hoffman, Ed., Oxford, England: Pergamon, 1960.

[17] T. Fawcett, "An introduction to ROC analysis," Pattern Recognition Lett., vol. 27, no. 8, pp. 861-874, June 2006.

[18] Wolfram, The Wolfram functions site. [Online] Available: http://functions.wolfram.com, 2009.

[19] A. Liu, E. F. Schisterman, and C. Wu, "Nonparametric estimation and hypothesis testing on the partial area under receiver operating characteristic curves," Commun. in Statistics - Theory and Methods, vol. 34, no. 9, pp. 2077-2088, Oct. 2005.

[20] M. K. Simon and M.-S. Alouini, Digital Communication over Fading Channels, 2nd ed. New York: Wiley, 2005. 
[21] A. Pandharipande and J. P. M. G. Linnartz, "Performance analysis of primary user detection in a multiple antenna cognitive radio," in Proc. IEEE Int. Conf. Commun. (ICC), June 2007, pp. 6482-6486.

[22] J. Ma, G. Zhao, and Y. Li, "Soft combination and detection for cooperative spectrum sensing in cognitive radio networks," IEEE Trans. Wireless Commun., vol. 7, no. 11, pp. 4502-4507, Nov. 2008.

[23] Y.-C. Liang, Y. Zeng, E. C. Y. Peh, and A. T. Hoang, "Sensingthroughput tradeoff for cognitive radio networks," IEEE Trans. Wireless Commun., vol. 7, no. 4, pp. 1326-1337, Apr. 2008.

[24] A. Annamalai and C. Tellambura, "Performance evaluation of generalized selection diversity systems over Nakagami- $m$ fading channels," Wireless Commun. and Mobile Computing, vol. 3, no. 1, pp. 99-116, July 2002.

[25] M. Gans, "The effect of Gaussian error in maximal ratio combiners," IEEE Trans. Commun. Technol., vol. 19, no. 4, pp. 492-500, Aug. 1971.

[26] B. R. Tomiuk, N. C. Beaulieu, and A. A. Abu-Dayya, "General forms for maximal ratio diversity with weighting errors," IEEE Trans. Commun., vol. 47, no. 4, pp. 488-492, Apr. 1999.

[27] G. G. Lorentz, Bernstein Polynomials, 2nd ed. New York: Chelsea Pub. Co., 1986.

[28] J. N. Pierce and S. Stein, "Multiple diversity with nonindependent fading," Proc. IRE, vol. 48, no. 1, pp. 89-104, Jan. 1960.

[29] A. P. Prudnikov, Y. A. Brychkov, and O. I. Marichev, Integrals and Series, vol. 2. Gordon and Breach science Publishers, 1986.

[30] I. S. Gradshteyn and I. M. Ryzhik, Table of Integrals, Series, and Products. New York: Academic Press, 2000.

[31] A. H. Nuttall, "Some integrals involving the $Q_{M}$ function," Naval Underwater Systems Center (NUSC), Tech. Rep., May 1974.

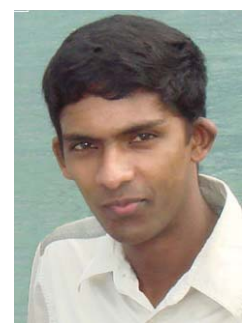

Saman Atapattu (S'06) received the B.Sc. degree in Electrical and Electronics Engineering (with a first class honor) from the University of Peradeniya, Sri Lanka in 2003 and the M. Eng. degree in Telecommunications from Asian Institute of Technology (AIT), Thailand in 2007. He is currently working towards the Ph.D. degree in Electrical Engineering at the University of Alberta, Canada. His research interests include cooperative communications, spectrum sensing in cognitive radio, energy detection and performance analysis of communication systems.

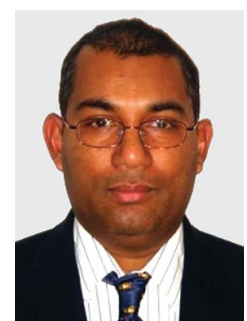

Chintha Tellambura (SM'02) received the B.Sc. degree (with first-class honor) from the University of Moratuwa, Sri Lanka, in 1986, the M.Sc. degree in Electronics from the University of London, U.K., in 1988, and the Ph.D. degree in Electrical Engineering from the University of Victoria, Canada, in 1993.

He was a Postdoctoral Research Fellow with the University of Victoria (1993-1994) and the University of Bradford (1995-1996). He was with Monash University, Australia, from 1997 to 2002. Presently, he is a Professor with the Department of Electrical and Computer Engineering, University of Alberta. His research interests include diversity and fading countermeasures, multiple-input multiple-output (MIMO) systems and space-time coding and orthogonal frequency division multiplexing (OFDM).

Prof. Tellambura is an Associate Editor for the IEEE TRAnSACTIONS ON COMMUNICATIONS and the Area Editor for Wireless Communications Systems and Theory in the IEEE TRANSACTIONS ON WIRELESS COMMUNICATIONS. He was Chair of the Communication Theory Symposium in Globecom'05 held in St. Louis, MO.

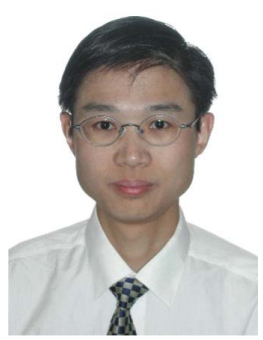

Hai Jiang (S'04-M'07) received the B.Sc. and M.Sc. degrees in electronics engineering from Peking University, Beijing, China, in 1995 and 1998, respectively, and the Ph.D. degree (with an Outstanding Achievement in Graduate Studies Award) in electrical engineering from the University of Waterloo, Waterloo, ON, Canada, in 2006.

Since July 2007, he has been an Assistant Professor with the Department of Electrical and Computer Engineering, University of Alberta, Edmonton, AB, Canada. His research interests include radio resource management, cognitive radio networking, and cross-layer design for wireless multimedia communications.

Dr. Jiang is an Associate Editor for the IEEE TRAnSACTIONS ON VEHICUlar Technology. He served as a Co-Chair for the General Symposium at the International Wireless Communications and Mobile Computing Conference in 2007, the Communications and Networking Symposium at the Canadian Conference on Electrical and Computer Engineering in 2009, and the Wireless and Mobile Networking Symposium at the IEEE International Conference on Communications in 2010. He received an Alberta Ingenuity New Faculty Award in 2008 and a Best Paper Award from the IEEE Global Communications Conference in 2008 . 